\title{
Mixed Mode Fracture in Concrete
}

\section{A. HILLERBORG}

Division of Building Materials, Lund Institute of Technology, Box 118, S-221 00 Lund, Sweden

\section{ABSTRACI}

The type of mixed mode fracture, which is of a main interest for concrete is a type of fractume, where shear as well as tensile stresses act in a damage zone, which has been created due to pure tensile stresses, and which is perpendicular to the dimection of these stresses. While this damage zone still transfers tensile stresses, a shear deformation takes place, which also causes shear stresses. A test set-up for the determination of the

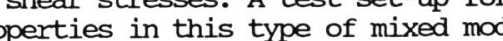
examples of results are given.

\section{KEYWORDS}

Fracture; mixed mode; shear; concrete; tests; properties.

\section{INIRODUCTION}

In linear elastic fracture mechanics the term "mixed mode" is used for the situation where the stress intensity factors $\mathrm{K}$ and $\mathrm{K}$ are both non-zero. The crack will grow in a direction, which is skew with respect to the direction of the existing crack.

For concrete structures of normal sizes another type of fracture mechanics is often applied, where stress intensity factors are not used, but stress-displacement relations for a damage zone. In a pure tensile fracture this can be described as strain-softening with strain localization.

Concrete is normally assumed to be an isotropic material with a much lower tensile strength than compressive strength. When the tensile strength is reached in such a material, a damage zone (sometimes formally described as a "fictitious crack" with cohesive forces) is formed perpendicular to the stress direction. The damage zone starts as separated microcracks, which coalesce into one single crack, often branched, before final failure. The direction of the damage zone (the "fictitious crack") in a point is determined by the direction of the tensile stress at the moment when the 
tensile strength is reached. When the deformation is further increased, the direction of the damage zone is as a rule maintained even if the stres direction changes. In such a case shear stresses as well as normal stresses will be acting in the damage zone. The shear stresses will be accompanied by shear displacements.

The definition of mixed mode, which will be used here, is a situation, where shear stresses as well as normal stresses are acting in a damage zone, which was originally fonmed perpendicular to a tensile stress direction, but where the stress direction has changed.
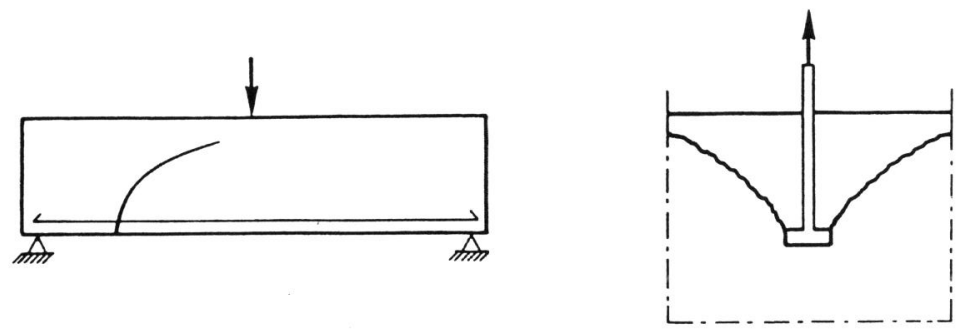

Fig. 1. Examples of damage zones with mixed mode fracture.

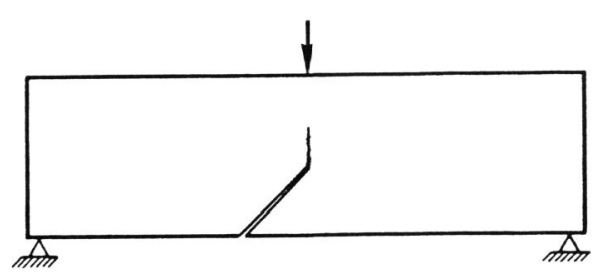

Fig. 2. Beam with a skew rotch and a damage zone.

Examples of situations, where this nay happen, are shown in Fig. 1. The trace of the damage zone which will develop into a crack, is as a rule curved in these cases.

From the point of view of conventional fracture mechanics the beam in Fig. 2 will be in a state of mixed mode. With the definition used here it will not be a mixed mode, as the stresses in the damage zone will all the time be practically perpendicular to the direction of this zone. Thus, the direction of the damage zone with respect to the direction of the notch has nothing to do with mixed mode.

In order to be able to make theoretical analyses of structures, where this type of mixed mode appears, it is necessary to know the relations between the stresses and displacements. The two stresses, normal and shear, both depend on the two displacements, nomal and shear, but also on how the displacements have been reached. The relations are thus path dependent. This complicates the problem of determining the relations by means of tests.

A series of tests will be described below, and some results will be given. The tests are being performed by M. Hassanzadeh, who has given a short description of the tests in some earlier papers (Hassanzadeh et al.' 1987, Hassanzadeh, 1988). A full report of the tests will soon be published.

\section{TEST SET-UP}

In the tests, a concrete specimen should be exposed to stress and displacement conditions, which as closely as possible correspond to those, which are expected in the structures, which are to be analysed. Thus in the beginning of the test a tensile stress shall create a well defined damage zone. After the damage zone has formed, the specimen shall be exposed to a combination of shear and normal displacements, which correspond to a reasonable path, which can be expected in a real structure.

In onder to achieve a well defined damage zone, and also to be able to In order the achieve aspecimen used in the tests has a notch all around defining the position of the damage zone, perpendicular to the normal stress, Fig. 3. The test area is $40 * 40 \mathrm{~mm}$

The displacement direction when the damage zone is formed is a pure normal displacement. When also a shear displacement starts to take place, the displacement direction will change gradually, as the damage zone grows. The relation between shear displacement and normal displacement is called the displacement path. It can be shown in a diagram. Figure 4 shows such a diagram with a number of different paths.

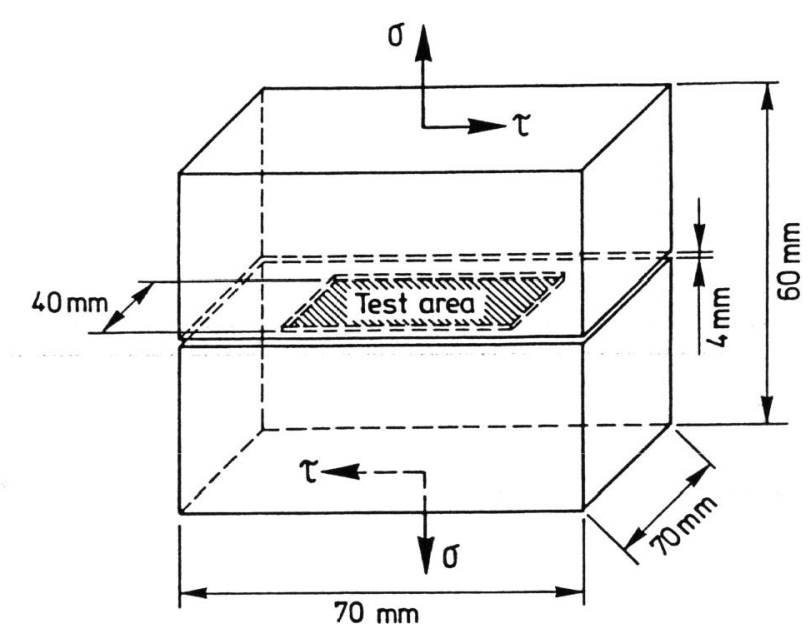

Fig. 3. Specimen used for the mixed mode tests. 


\section{Normal displacement}

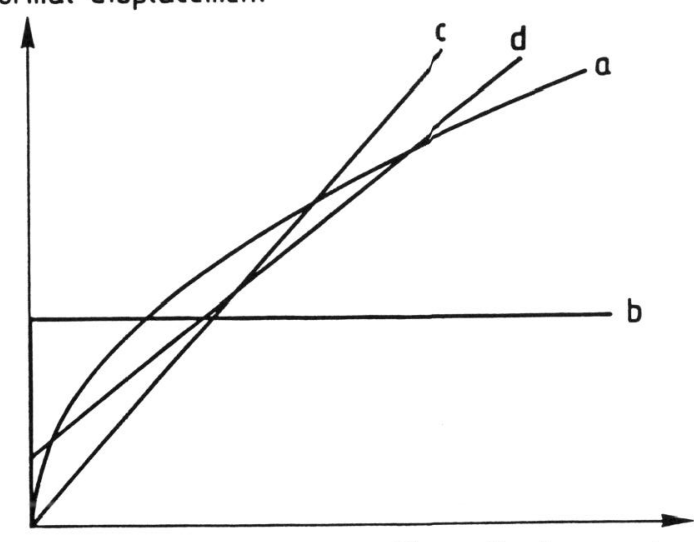

Shear displacement

Fig. 4. Examples of displacenents paths.

A realistic path with regard to application to real structures is such, that it starts with a pure normal direction, i.e. a vertical direction at the origin, and gradually changes its direction. Thus curve a) is realistic, upon as an approximation of a) by mears of straight lines.

For the main test series a displacemert path of type a) in Fig. 4 has been used in order to achieve realistic results. As shape of the curve a second degree parabola has been chosen. Different parabolas have been used. Some dependency.

A problem with tension tests in order to find the stress-displacement relation is the risk of instability. Two types of instabilities have to be taken into account.

The first type of instability, which is very evident, is the stability with regard to rate of change of normal displacement and the corresponding tensile stresses. In a stable test no sudden jumps in normal displacement may take place. This problem can be overcome by means of modern closed-loop testing machines. Such a machine has keen used to impose the normal displacements.

The second type of instability, which is less well known, is a bending or rotational instability, giving rise to an uneven tensile stress distribution in the damage zone. The instability is of the same type as buckling of a column. The descending stress-displacement relation for the damage zone corresponds to a negative modulus of elasticity. A negative modulus of elasticity in a structure with tensile forces is equivalent to a positive
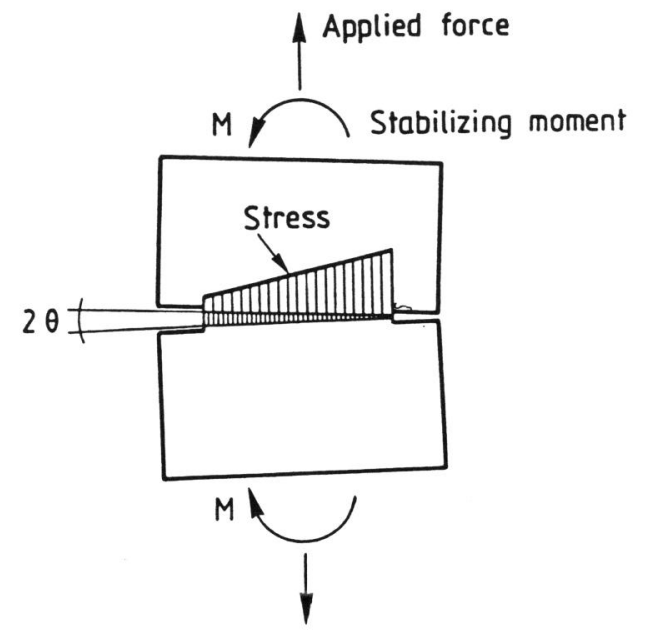

Fig. 5. Rotation in a damage zone.

modulus of elasticity in a structure with compressive forces when it comes to stability criteria.

The stabilizing moments, which prevent the instability in tension failure, The stabilizing me test equipment have to come from the test equipment. The stiffness of the test equipr must be high enough to prevent the ro in (Hassanzadeh et al., 1987). It can stability criterium has been studied

$$
\alpha>-2 \mathrm{Id} \sigma / \mathrm{d} w
$$

here $\alpha$ is the stiffness on each side of the damage zone, I is the moment of inertia of the damage zone section, and $\mathrm{d \sigma} / \mathrm{dw}$ is the slope of the

stress-displacement diagram in tension (which is always negative). The stabilizing moment $M$ on one side of the damage zone, and the corresponding angle of deformation $\theta$, Fig. 5.

$$
\alpha=\mathrm{M} / \mathrm{\theta}
$$

In order to make the test completely stable, the above condition must be fulfilled also for the steepest part of the $\sigma-w$ diagram, which normally is in the beginning. If the stiffness is too low, the stress distribution will be uneven at high stresses, but get more even as the stresses decrease. The necessary stiffness for a completely stable test is very high, and it is difficult to fulfill the condition, particularly when the test equipment shall allow also shear displacements.

The solution, which has been chosen to solve this problem, is to insert the specimen between two stiff beams, which are connected at one end in a way which prevents torsional rotation, but not the displacement corresponding to shear displacement in the specimen. The test arrangement is shown in Fig. 6. 


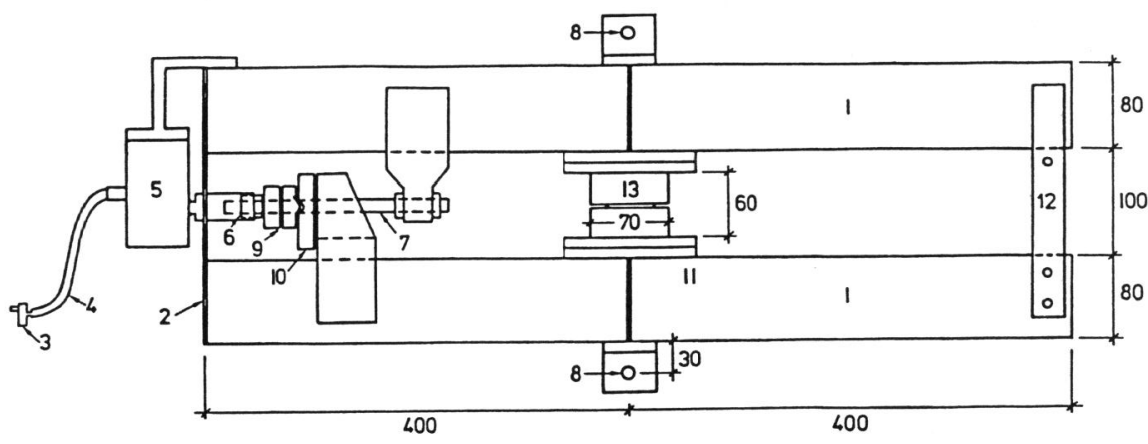

1- Box Beams (hight $=80 \mathrm{~mm}$, width $=40 \mathrm{~mm}$, thickness $=3 \mathrm{~mm}$ ) 2- Steel sheet (hight $=260 \mathrm{~mm}$, width $=100 \mathrm{~mm}$, thickness $=1 \mathrm{~mm}$ ) 3 - Crank

4- Flexible axle

5 - Gear box

6 - Nut

7 - Steel bar with strain gauges

8 - Connection point to testing machine

9 - Roller support

10- Slip support

11 - Steel plates

12 - Lock (it is removed during the test

13- Specimen

Fig. 6. Test arrangement.

With this arrangement the normal displacement is not quite even over the damage zone, as the beams perform a rotational movement around their right ends. This unevenness can however be shown to be of a negligible importance, of the test equipment (Hassanzadeh et al., 1987).

The normal displacement is achieved by means of a MIS testing machine with a closed loop system, which gives the stability in the normal direction. The closed loop system, which gives the stability in the normal a screw

arrangement bentwe

normal displetween the two beams, which is shown in fisplacement rate is

varied in order to make the displacenent path follow the predetermined

shape, cf. Fig. 4.
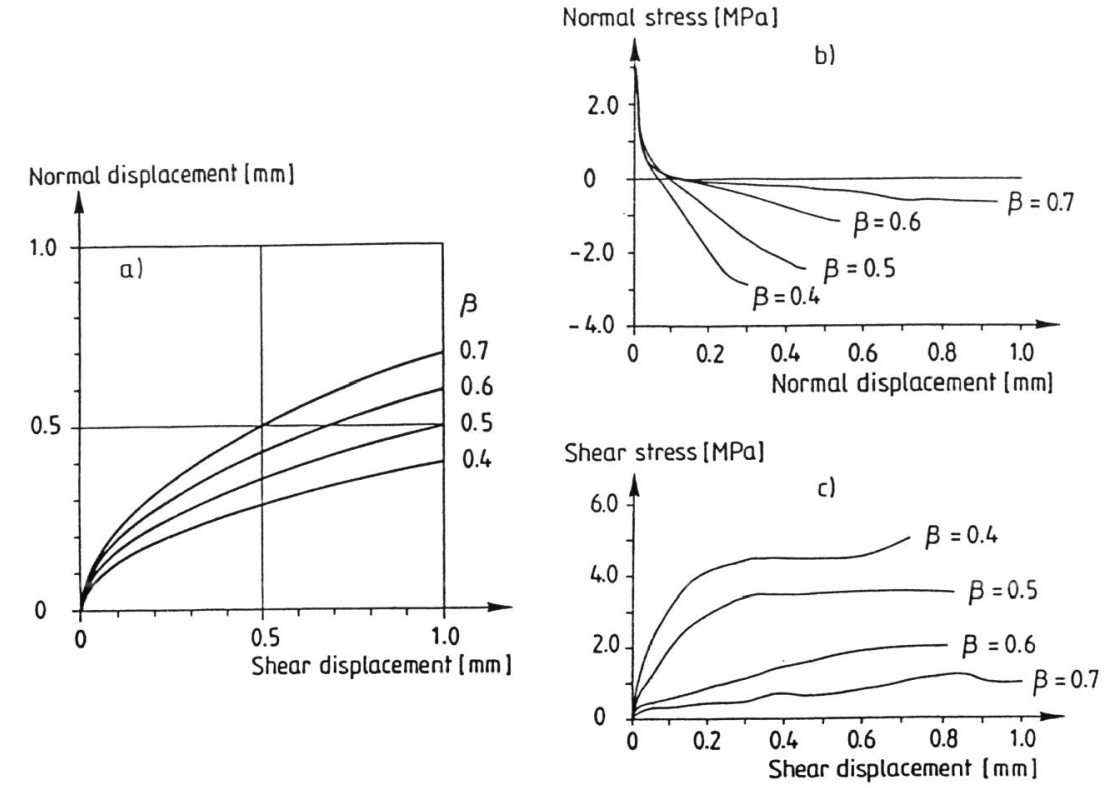

Fig. 7. Test results.

TEST RESULTS

For the reasons mentioned above, the main test series has been made with a parabolic displacement path. This can be described by means of the equation

$$
\mathrm{w}_{\mathrm{n}}=\beta \sqrt{\mathrm{w}}_{\mathrm{S}}
$$

where $\mathrm{w}$ is the normal displacement, and $\mathrm{w}$ is the shear displacement. Figure $\mathrm{Ya}$ shows displacement paths used in the tests, with different values of $\beta$ in $\mathrm{mm}^{2}$. Figures $7 \mathrm{~b}$ and $\mathrm{c}$ show the corresponding measured stress displacement curves. The tested concrete had a water cement ratio of 0.5 , and a maximum particle size of $8 \mathrm{~mm}$. It was tested in a wet condition at an age of 28 days.

Some tests have also been made with a displacement path according to c) in Fig. 4 in order to study the path dependency (Hassanzadeh, 1988). These tests showed a clear path dependency. The stress at a given deformation reached along paths a) and c) differed at the most with a factor of about 2 Taking into account that path c) is rather unrealistic, this difference is however not very large. Possibly curve b) would have shown larger differences. The preliminary conclusion from these tests is that the path dependency is not very important, as long as the paths lie within realistic limits.

It can be seen from Fig. 7 that the value of $\beta$, i.e. the displacement path, has a great influence on the stress-displacement curves. For values of $\beta$ down to about 0.7 the shear stresses are small, and the stress-displacement 
curve for normal stresses is rather unaffected. For low values of $\beta$ the shear stresses dominate, and the normal stresses become compressive, with relatively high values. The behaviour in this case can be explained in two different ways.

One possible way of explaining the behaviour is by means of the microcracks, which start as very small tensile cracks, but bend off and change direction as shear forces start acting. Thus they grow in a skew direction according to Fig. 8. In this way a system of skew compression struts are formed, which perform a rotating movement, which gives rise to compressive and shear stresses while the normal and shear displacements increase.

Another possible explanation is the irregular shape of the damage zone, Fig 9. Due to the inhomogenous structure of concrete, the damage zone is not straight, but has an irregular shape. When a shear displacement occurs, friction and compressive forces are caused by these irregularities. This is the same type of behaviour which has been studied for stresses in a real crack (Paulay and Loeber, 1974, Walraven, 1980).

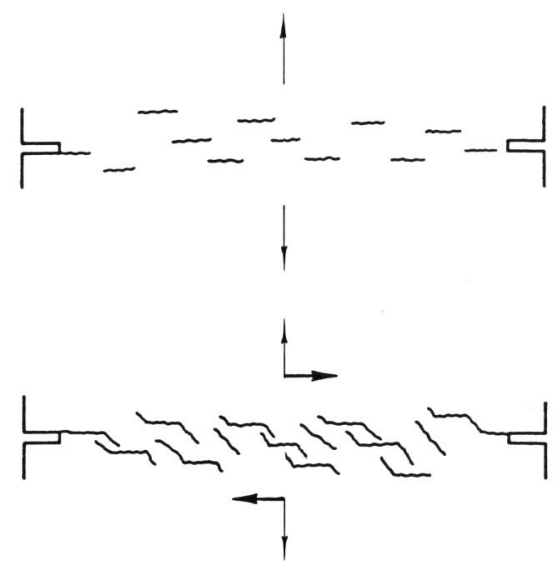

Fig. 8. Development of nicrocracks in mixed mode.

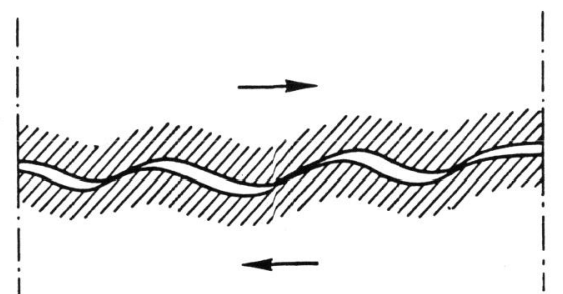

Fig. 9. Influence of irregularities in a crack with a shear displacement. of these two explanations, the first one seems most probable for the early stages, when the microcracks are still small, while the latter explanation may be more relevant at the later stages, when the shear displacement is relatively large.

The results shown in Fig. 7 are of course only valid for the tested concrete. Further tests are needed in order to study how the properties depend on the concrete composition.

In fracture mechanics of concrete the fracture energy is often used as a parameter. It is a suitable parameter for the case of a pure opening type of fracture, with only tensile stresses in the damage zone during the whole fracture process. In mixed mode the situation is much more complex, as neroy is also absorbed in shear stresses and displacements. From Fig. 7 it can be seen that this energy absorption (areas under the curves) is dominating for small values of $\beta$, and it also varies much with $\beta$. Thus the fracture energy cannot be used as a material parameter in the case of mixed mode fracture. So far the unly way to describe the fracture properties in mixed mode fracture seen the type shown in Fig. 7

For small values of $\beta$ the shear stresses become rather high, see Fig. 7 . From the shear and normal stresses in the damage zone, and the normal stresses parallell to the damage zone, the total two-dimensional stress state can be calculated. This stress state is also valid for the undamaged material close to the damage zone. The principal tensile stress outside the damage zone may then lead to the formation of a new damage zone at a skew angle to the first one. It is possible that a crack, which is visible at early stage, at a later stage is crossed by a new crack at a skew angle, which often becomes the dominating crack, leading to the final failure. Such a behaviour can often be noticed in shear failure of reinforced concrete beams.

\section{CONCLUSIONS}

structures is best described as combination of normal stresses and shear stresses in a damage zone, which has started due to pure tensile stresses.

The normal and shear stresses both depend on normal displacement, shear displacement, and the displacement path.

In onder to achieve realistic relations between stresses and displacements, to be used in a numerical analysis, the displacement path used in the test must not be too different from the path found in the analysis. The path dependency does however not seem to be strong, as long as the type of path is reasonably realistic.

The demand on stiffness of the testing equipment is very high in order to avoid a rotational instability in the damage zone, which may cause severe errors in the measured properties. 
REFERENCES

Hassanzadeh, M., Hillerborg, A. and Zhou, F.P. (1987). Test of material properties in mixed mode I and II. International conference on Fracture of Concrete and Rock, Houston, Texas, June 17-19, 1987.

consadeh M. (1988). Determination of fracture zone properties in mixed mode I and IT. International Conference on Fracture of concrete ar Vienna, July 4-6, 1988 .

Ioeber, P.S. (1974). Shear transfer by aggregate interlock.

Shear in reinforced concrete, ACI SP-42, Vol.1, 1-15.

J.C. (1980) Angregate interlock: a theoretical and experimental analysis. Dissertation, Delft University of Technology. 